\title{
A framework for modelling cash flow lags
}

\author{
Fredrik Armerin $^{1}$ (D) Han-Suck Song ${ }^{1}$
}

Received: 7 May 2021 / Accepted: 23 August 2021 / Published online: 27 September 2021

(C) The Author(s) 2021

\begin{abstract}
Traditional models of irreversible investment problems assume that the investment starts generating cash flows immediately, i.e., at the same time as the investment is undertaken. Real-world investment situations are characterized by time-to-build or investment lags, which means that there is a time difference between when the investment is made and when the investment starts generating cash flows. We combine two existing models of investment lags to obtain a flexible, yet simple, way of modelling and analyzing the effects of investment lags. Both traditional models, and models that incorporate the effects of time-to-build, typically assume that the expected future cash flows generated by an investment are represented by a single cash flow that reflects the size of the market value of an investment. To reflect realworld cases where investments generate cash flows in several time periods, we present a framework in which cash flows are explicitly allowed to be spread out in time. Our model can be used to incorporate cases where an investment is partially sold in different time periods. Using an irreversible optimal investment timing problem case study, we show how our framework makes it possible to easily compare the effect of different cash flow timings. In this case, the value and the timing of the investment depend on a constant that in a natural way can be decomposed into three parts, thereby showing the influence of the value and timing from the respective parts of the framework.
\end{abstract}

Keywords Optimal stopping · Irreversible investments · Cash flow lags · Time-tobuild

JEL Classification $\mathrm{G} 11 \cdot \mathrm{G} 13 \cdot \mathrm{R} 30$

Fredrik Armerin

fredrik.armerin@abe.kth.se

Han-Suck Song

han-suck.song@abe.kth.se

1 Department of Real Estate and Construction Management, KTH Royal Institute of Technology, Stockholm, Sweden 


\section{Introduction}

Traditional models of irreversible investment problems studied in finance has the property that the investment starts generating cash flows immediately, i.e., at the same time as the investment is undertaken. For instance, in the standard optimal timing investment problem, as presented in the seminal paper by McDonald and Siegel (1986), and in the classical (Dixit and Pindyck 1994) textbook, it is assumed that the entire fixed investment cost is paid at the time the investment is undertaken, and that the investment starts generating net cash flows immediately, that is, at the same as time the investment is undertaken.

Mathematically, the problem for the investor to solve is

$$
\sup _{\tau} E^{Q}\left[e^{-r \tau}\left(X_{\tau}-I_{\tau}\right)\right]
$$

Here $\tau$ is the time of the investment, $I_{\tau}$ is the cost of the investment, $X_{\tau}$ is the present value of cash flows generated by the investment (McDonald and Siegel 1986), $r$ is the risk-free interest rate and $Q$ is the risk-neutral measure; see below for details. Without essential loss of generality, we will from now on assume that the cost is a fixed constant $I>0$. In this case the optimal investment problem is an example of an optimal stopping problem on the form

$$
\sup _{\tau} E^{Q}\left[e^{-r \tau} G\left(X_{\tau}\right)\right]
$$

In real-world situations, it takes time before an investment starts generating cash flows, that is when an investment starts generating revenues and costs. The time difference between the time the investment outlays are paid (or investment decisions are made) and the time the investment starts to generate cash flows is referred to as time-to-build, construction lag, gestation period, investment delay, investment lag or delivery lag (see e.g. Bar-Ilan and Strange 1996; Lempa 2012).

Investment delays can span over many years. Pacheco-de-Almeida and Zemsky (2003) refer to an empirical study by Koeva (2000) who measures the average timeto-build in 23 industries. Koeva (2000) finds, for instance, that the investment lag for rubber processing plants is 13 months, 23 months for chemical plants and 86 months for utilities industry. Large scale real investment projects such as an investment in an office building, or R\&D projects aiming at commercialization of new technologies, can range from months to years (Lempa 2012).

Since the introduction of the traditional models where time-to-build and investment delays were assumed to not exist, various investment models have been developed that analyze the effects of time-to-build. Examples of early models of timeto-build include (Bar-Ilan and Strange 1996; Alvarez and Keppo 2002; Øksendal 2005; Lempa 2012) and (Sarkar and Zhang 2013). Examples of other types models where time lags are present include Aguerrevere (2003), Bar-Ilan, Sulem \& Zanello (2002), Grenadier (1995), Grenadier (2000), Kalouptsidi (2014), Majd and Pindyck (1987) and Margsiri et al. (2003) (the same model for the time-to-build is used in Sarkar and Zhang 2015). For a non-technical discussion on real options and investment lags, see MacDougall and Pike (2003). Lempa (2020) represents a recent 
theoretical work on implementation delays. We combine two different models of investment lags to obtain a flexible, yet simple, way of modelling and analyzing the effects of investment delays.

Marmer and Slade (2018) is an empirical study on the effect of investment delays of entry into U.S. copper mining, and they argue that investment delays should be given more attention in theoretical and empirical work. There exist several examples of recent literature that study the effect of investment lags in various industries. Oh and Yoon (2020) study how construction lags affect decisions around residential construction projects. Balliauw (2020) considers time-to-build in port capacity expansion investments. Jeon (2021a) studies a firm's investment, default, and financing decisions in the presence of time-to-build, and Jeon (2021) studies timeto-build affects the decisions of investment timing and capacity in a duopoly market. Taschini (2021) studies investment in abatement technology and emission trading system that are characterized by significant implementation lags.

Furthermore, both traditional models, and models that incorporate the effects of time-to-build, typically assume that the expected future cash flows generated by an investment are represented by a single cash flow that reflects the size of the market value of an investment (e.g. a factory, a real estate project or share of stocks in a venture capital investment). To better reflect real-world cases where an investment can generate cash flows in several time periods, we also present a framework in which cash flows generated by an investment are explicitly allowed to be spread out in time. Our model can for instance be used to incorporate cases where an investment is partially sold in different time periods (e.g. partial selling buildings in a multibuilding real estate projects or selling of a certain percentage of initial investments in share of stocks in venture capital investment). Or simply, our model can reflect the actual net cash flows (cash inflows and cash outflows) an investment generate.

Øksendal (2005), Alvarez and Keppo (2002), Lempa (2012), and Sarkar and Zhang (2013) represent previous implementation delay modelling studies that we aim to generalize to also allow for more general cash flow lags. The recent paper Lempa (2020) extends the model developed in Lempa (2012) from an exponentially distributed implementation delay to a general phase-type distributed implementation delay. We, on the other hand, essentially take the model in Lempa (2012) and extend it to where cash flows are spread out in time after the implementation delay has passed. Indeed, in this paper we focus on extending mainly the class of models given above to where the cash flows are spread out in time after the investment is starting to generate cash flows. In many cases, this property is prevalent, and allowing models to include it will increase the precision in the results of a model.

Mathematically, the main structure of the modelling framework is as follows: After the time $\tau$ at which the investment is initiated, and where there can be a cash flow, there passes a random time $\tau_{B}$ during which there are no cash flows (this could e.g. be the time-to-build in a real estate project, or the time until a start-up company is beginning to generate cash flows, sell assets or a certain percentage of share of stocks owned). To model the distribution of $\tau_{B}$, we use a combination of two models: The one given in Øksendal (2005) (who uses a constant time) and the one given in Lempa (2012) (who uses an exponentially distributed time). In this way we get a flexible, yet simple, way of modelling the cash flow lag. After the time $\tau+\tau_{B}$, the 
investment is starting to generate cash flows. The unique property of our approach is that there can be cash flows during the interval $\left[\tau+\tau_{B}, \infty\right)$, not only at time $\tau+\tau_{B}$. By using this framework, we can gain a better understanding on how the different parts of the model influence the time at which the investment is initiated, as well as the value of the investment.

It is well known that in problems such as the optimal investment problem described above, it is optimal to wait longer than to the first time until $X_{t}-I$ is positive; this difference needs to be large enough before it is optimal to initiate the investment. When there is an extra delay imposed exogenously, the optimal time of investment can be both earlier (as in Bar-Ilan and Strange 1996) and later (as in the case study in Section 3 below) than in the case without the delay in cash flows.

The rest of this paper is organized as follows. In Section 2 the modelling setup of previous approaches to investment lags as well as our extension of existing models are presented, Section 3 contains an application of our modelling framework, and Section 4 concludes.

\section{Modelling cash flow lags}

Let $\left(X_{t}\right)$ be a strong Markov process defined on a suitable probability space. We also assume the existence of a risk-neutral probability measure $Q$, locally equivalent to the original probability measure, such that the value of a future uncertain cash flow is given by the expected value using this measure and discounting the cash flow using the constant bank account rate $r>0$. See e.g. (Jeanblanc et al. 2009) for the underlying theory.

Now consider the following class of investment problems. At time $\tau$, at which the investment is initiated, there can be a lump sum cash flow. Then there is a time span $\tau_{B}$ under which there are no cash flows. The time $\tau_{B}$ could e.g. be the time-to-build in a real estate project, or the time until a start-up company is beginning to generate cash flows. At time $\tau+\tau_{B}$ there can also be a lump-sum payment. The investor's goal is to maximize the total value of these cash flows, i.e. to solve the problem

$$
\sup _{\tau} E_{x}^{Q}\left[e^{-r \tau} G\left(X_{\tau}\right)+e^{-r\left(\tau+\tau_{B}\right)} G_{B}\left(X_{\tau+\tau_{B}}\right)\right] .
$$

Here $G\left(X_{\tau}\right)$ is the lump sum cash flow at time $\tau$ and $G_{B}\left(X_{\tau+\tau_{B}}\right)$ is the lump sum cash flow at time $\tau+\tau_{B}$. The notation $E_{x}^{Q}$ means that we consider the expected value under $Q$ given that $X_{0}=x$.

Example 1 Several of the models mentioned in the Introduction fits into the modelling setup described in Eq. (2).

(i) McDonald and Siegel (1986): $G(x)=x-I, \tau_{B}=0$ and $G_{B}(x)=0$.

(ii) Øksendal (2005): $G(x)=0, \tau_{B}=\delta \in \mathbb{R}_{+}$and $G_{B}$ is a general function.

(iii) Alvarez and Keppo (2002): $G(x)=I, \tau_{B}=\Delta\left(X_{\tau}\right) \geq 0$ with $\Delta(0)=0$ and $G_{B}(x)=x$. 
(iv) Lempa (2012): $G(x)=0, \tau_{B}$ is independent of $X$ and exponentially distributed and $G_{B}$ is a general function.

(v) Margsiri et al. (2003): $G(x)=\theta(x-I), \tau_{B}$ a hitting time of $X$ and $G_{B}(x)=(1-\theta) \cdot(x-I)$, where $\theta \in(0,1)$.

(vi) Lempa (2020): $G(x)=0, \tau_{B}$ is independent of $X$ and has a phase-type distribution and $G_{B}$ is a general function.

It should be noted that using the (strong if necessary) Markov property, all cases in the previous example can be reduced to a problem on the form given in Eq. (1); see the referred literature for details in the respective case.

In practise, the cash flows occurring after the time $\tau+\tau_{B}$ are not restricted to one lump sum payment, typically representing the market value (present value) of future cash flows, but are in general spread out over time. The same may be true of the cash flows occurring at time $\tau$, but here we focus on the cash flows after time $\tau+\tau_{B}$ (generalising to also include the other case is straightforward). In order to allow for cash flows at times $s$ after the time $\tau+\tau_{B}$, we consider optimal stopping problems on the following form:

\section{Problem (P)}

$$
\sup _{\tau} E_{x}^{Q}\left[e^{-r \tau} G\left(X_{\tau}\right)+e^{-r\left(\tau+\tau_{B}\right)} \int_{0}^{\infty} e^{-r s} G_{B}\left(X\left(\tau+\tau_{B}+s\right)\right) \mathrm{d} F(s)\right] .
$$

When using this approach to model investments, $\tau$ is the time at which it is decided that the investment should be initiated, $G\left(X_{\tau}\right)$ the lump sum cash flow at this time, $\tau+\tau_{B}$ is the time at which further cash flows from the investment is starting to appear and $F$ is a probability measure on $[0, \infty)$ describing how the cash flows are distributed after the time $\tau+\tau_{B}$. The value of $s$ measures the distance in time from $\tau+\tau_{B}$.

\section{Example 2}

(i) Lump sum payments. The cash flow(s) occur at given time spans $t_{1}, \ldots, t_{n}$ after the time $\tau+\tau_{B}$ :

$$
F(s)=\sum_{i=1}^{n} w_{i} \delta_{t_{i}}(s)
$$

Here the $w_{i}$ 's are strictly positive and sum to 1 and $\delta_{x}$ denotes the Dirac measure at $x$ (here we only consider $x \in \mathbb{R}_{+}$). In this case the optimal stopping Problem $(\mathrm{P})$ can be written

$$
\sup _{\tau} E_{x}^{Q}\left[e^{-r \tau} G\left(X_{\tau}\right)+e^{-r\left(\tau+\tau_{B}\right)} \sum_{i=1}^{n} w_{i} e^{-r t_{i}} G_{B}\left(X\left(\tau+\tau_{B}+t_{i}\right)\right)\right] .
$$


The choice $w_{1}=1$ and $F(s)=\delta_{0}(s)$ represents the optimal stopping problem in Eq. (2).

(ii) Continuous payouts. In this case $d F(s)=f(s) d s$ for some density function $f$ on $[0, \infty)$. The optimal stopping problem can now be written

$$
\sup _{\tau} E_{x}^{Q}\left[e^{-r \tau} G\left(X_{\tau}\right)+e^{-r\left(\tau+\tau_{B}\right)} \int_{0}^{\infty} e^{-r s} G_{B}\left(X\left(\tau+\tau_{B}+s\right)\right) f(s) \mathrm{d} s\right] .
$$

To get a tractable, yet general, model we consider time delays $\tau_{B}$ that are the sum of a random time $U$ independent of the process $X$ and exponentially distributed with mean $1 / \lambda$, and a constant time delay $\delta \geq 0$ :

$$
\tau_{B}=\delta+U
$$

Hence, we combine the models of Øksendal (2005) and Lempa (2012), and extend it to cash flows spread out in time after the time $\tau+\tau_{B}$ has occurred.

One feature of this model is that it is in fact on the form given in Eq. (1), and this makes the suggested framework tractable from a computational point of view. In the following proposition we use the standard operators $P_{t}$ for $t \geq 0$ and $R_{\lambda}$ for $\lambda>0$ defined by

$$
P_{t} f(x)=E_{x}^{Q}\left[f\left(X_{t}\right)\right]
$$

and

$$
R_{\lambda} f(x)=E_{x}^{Q}\left[\int_{0}^{\infty} e^{-\lambda s} f\left(X_{s}\right) \mathrm{d} s\right]=\int_{0}^{\infty} e^{-\lambda s} P_{s} f(x) \mathrm{d} s
$$

respectively, and where we assume that each $f$ used is such that the respective operator is well defined.

Proposition 1 With notation and assumptions as above, and with a $\tau_{B}$ on the form in Eq. (3), Problem (P) can be written

$$
\sup _{\tau} E_{x}^{Q}\left[e^{-r \tau} H\left(X_{\tau}\right)\right]
$$

where

$$
H(x)=G(x)+\lambda e^{-r \delta} P_{\delta} R_{r+\lambda} G_{B}^{F}(x)
$$

and

$$
G_{B}^{F}(x)=\int_{0}^{\infty} e^{-r s} E_{x}^{Q}\left[G_{B}\left(X_{s}\right)\right] \mathrm{d} F(s) .
$$

The proof can be found in Online Resource 1 . 


\section{An investment case study}

We now present a concrete model of the cash flows generated by the investment, as well as of the investment problem. Under the pricing measure $Q$, the cash flow process $X$ is assumed to be a geometric Brownian motion:

$$
\mathrm{d} X_{t}=(r-q) X_{t} \mathrm{~d} t+\sigma X_{t} \mathrm{~d} W_{t}^{Q} .
$$

Here $q>0$ is the constant yield or implied yield (see Armerin and Song 2018 for a discussion), $\sigma>0$ is the volatility and $W^{Q}$ is a standard Wiener process under $Q$. At the inception of the investment, the constant $\cos t I>0$ is paid, i.e.

$$
G(x)=-I \text {. }
$$

All cash flows after time $\tau+\tau_{B}$ are simply cash flows that the investment generates, which means that

$$
G_{B}(x)=x,
$$

and finally the revenue cash flows are distributed according to the deterministic distribution function $F$ after the random time $\tau+\tau_{B}$. Hence, the optimal investment problem is to find the function

$$
V(x)=\sup _{\tau} E_{x}^{Q}\left[-e^{-r \tau} I+e^{-r\left(\tau+\tau_{B}\right)} \int_{0}^{\infty} e^{-r s} X\left(\tau+\tau_{B}+s\right) \mathrm{d} F(s)\right],
$$

and an optimal stopping time $\tau^{*}$. We show in Online Resource 1 that the value function $V$ is given by

$$
V(x)= \begin{cases}\left(V_{c}-I\right)\left(\frac{x}{k V_{c}}\right)^{a} & \text { when } x<k V_{c} \\ \frac{x}{k}-I & \text { when } x \geq k V_{c}\end{cases}
$$

where

$$
\begin{aligned}
& k=\frac{e^{q \delta}(1+q / \lambda)}{\int_{0}^{\infty} e^{-q s} \mathrm{~d} F(s)}, \\
& V_{c}=\frac{a}{a-1} I
\end{aligned}
$$

and

$$
a=\frac{1}{2}-\frac{r-q}{\sigma^{2}}+\sqrt{\left(\frac{1}{2}-\frac{r-q}{\sigma^{2}}\right)^{2}+\frac{2 r}{\sigma^{2}}}>1 .
$$

Furthermore, an optimal stopping time is given by

$$
\tau^{*}=\inf \left\{t \geq 0 \mid X_{t} \geq k V_{c}\right\}
$$


The factor $k$ is always greater than or equal to 1 , so the result of the delay in cash flows is that the optimal level at which the investment should be done is increased compared to the non-delayed case. This in turn will lead to a delay in the time of investment. Even in the case where there is no time-to-build (this is represented by the case $\delta=0$ and $\lambda=\infty$ ), there will in general be a delay in the time at which the investment is done due to the fact that the cash flows occur later in time. Note that we can write

$$
k=e^{q \delta} \cdot(1+q / \lambda) \cdot \frac{1}{\int_{0}^{\infty} e^{-q s} \mathrm{~d} F(s)} .
$$

Hence, there is a natural multiplicative decomposition of the constant $k$. The first term refers to the deterministic time delay, the second to the random time delay, and the third to the lag in cash flows.

It is clear that the difference between the value of the investment problem without any delays in the cash flows, and the case we study here, is captured by the value of the constant $k$. Writing $V(x ; k)$ for the value function with parameter value $k$, we note the important property that

$$
k_{1} \geq k_{2} \Rightarrow V\left(x ; k_{1}\right) \leq V\left(x ; k_{2}\right) \text { for all } x \geq 0 .
$$

In Figure 1, the circled points represents the lowest value for which it is optimal to invest. Note that the value of the function $V$ at this point is same irrespectively of the value of the parameter $k$ :

$$
V\left(k V_{c}\right)=V_{c}-I .
$$

To study how the parameters influence the value of $k$, we start by noting

$$
\frac{\partial k}{\partial \delta}=q k>0
$$

and

$$
\frac{\partial k}{\partial \lambda}=-\frac{q}{\lambda(\lambda+q)} k<0
$$

As the constant waiting time $\delta$ increases, the cash flows are occurring further away in the future, implying a higher value of $k$, and a smaller value function. Since the average time we have to wait after the constant waiting time $\delta$ is equal to $1 / \lambda$, an increase in $\lambda$ shortens this waiting time, resulting in a lower value of $k$, and hence of a larger value function.

The dependence of $k$ with respect to the yield (or implied yield) $q$ is given by

$$
\frac{\partial k}{\partial q}=\left(\delta+\frac{1}{\lambda+q}+\frac{\int_{0}^{\infty} s e^{-q s} \mathrm{~d} F(s)}{\int_{0}^{\infty} e^{-q s} \mathrm{~d} F(s)}\right) k>0 .
$$

The sign is due to the fact that as long the investment is not initiated, we can not enjoy the cash flows given by the yield. For an interpretation of this when there 


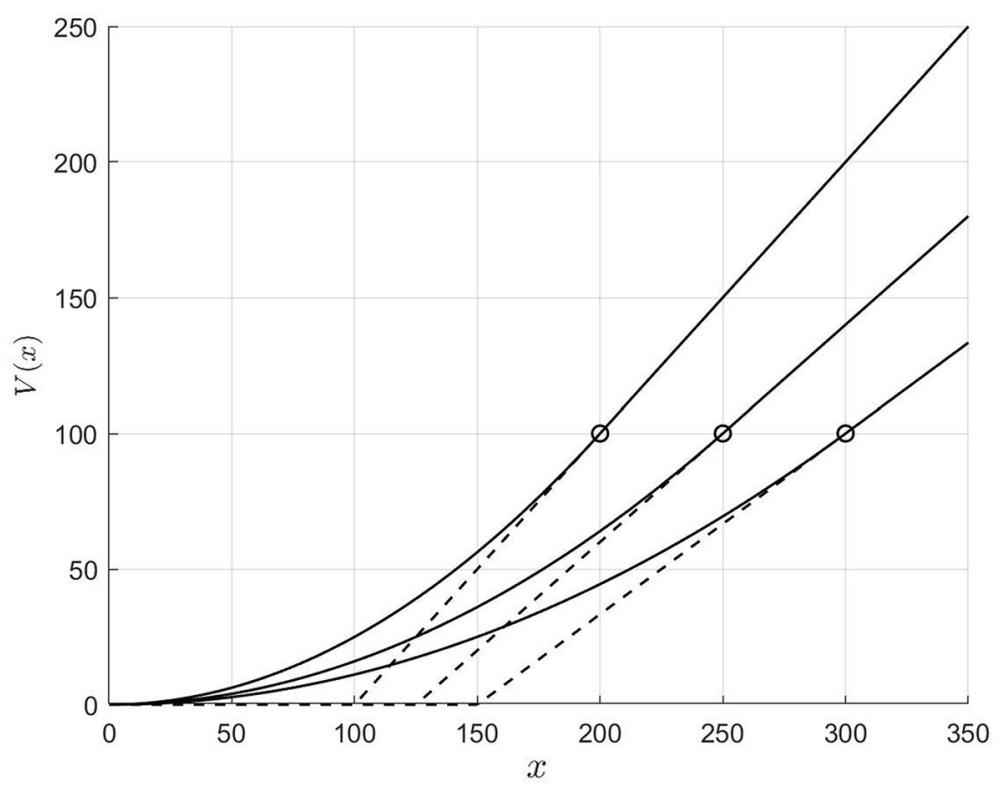

Fig. 1 The parameter values are $I=100$ and $a=2$, which gives $V_{c}=200$. The dashed lines are the gain functions and the solid lines are the values in the three cases (from left to right) $k=1, k=1.25$ and $k=1.5$ respectively. The circled points represents the lowest value at which it is optimal to invest

is only an implied yield, see the discussion on incomplete models in Armerin and Song (2018). We note that the last term in the parentheses in Eq. (5) can be interpreted as a duration, where the cash flows are distributed according to the function $F$, and there is a constant discount rate $q$.

Finally, we study how the distribution $F$ of cash flows influence the value of $k$, while keeping all other parameters constant. This analysis is slightly more technical than the previous one, and we refer to Online Resource 1 for definitions and proofs. We have

$$
F_{1}(x) \geq F_{2}(x) \text { for every } x \in[0, \infty) \Rightarrow \frac{1}{\int_{0}^{\infty} e^{-q s} \mathrm{~d} F_{1}(s)} \leq \frac{1}{\int_{0}^{\infty} e^{-q s} \mathrm{~d} F_{2}(s)} .
$$

The condition $F_{1}(x) \geq F_{2}(x)$ for every $x \in[0, \infty)$ in our context means that when the cash flows are distributed according to $F_{2}$, they are further away than when distributed according to $F_{1}$. It follows that we get a higher $k$ when the cash flows are distributed according to $F_{2}$ compared to when they are distributed according to $F_{1}$, and thus a smaller value function $V$ in the first case $\left(F_{2}\right)$ compared to the second case $\left(F_{1}\right)$.

Now, $F_{1}(x) \geq F_{2}(x)$ for every $x \in[0, \infty)$ is the definition that the distribution with distribution function $F_{2}$ first-order stochastically dominates the distribution with distribution function $F_{1}$, written $F_{1} \preceq_{\mathrm{FSD}} F_{2}$. Hence, we can rephrase the previous result as 


$$
F_{1} \preceq_{\mathrm{FSD}} F_{2} \Rightarrow \frac{1}{\int_{0}^{\infty} e^{-q s} \mathrm{~d} F_{1}(s)} \leq \frac{1}{\int_{0}^{\infty} e^{-q s} \mathrm{~d} F_{2}(s)} .
$$

In fact, the following stronger result holds:

$$
F_{1} \preceq_{\mathrm{SSD}} F_{2} \Rightarrow \frac{1}{\int_{0}^{\infty} e^{-q s} \mathrm{~d} F_{1}(s)} \leq \frac{1}{\int_{0}^{\infty} e^{-q s} \mathrm{~d} F_{2}(s)} .
$$

Here, $F_{1} \preceq_{\mathrm{SSD}} F_{2}$ means that $F_{2}$ second-order stochastically dominates the distribution with distribution function $F_{1}$, and the result is stronger since

$$
F_{1} \preceq_{\mathrm{FSD}} F_{2} \Rightarrow F_{1} \preceq_{\mathrm{SSD}} F_{2} .
$$

For a numerical example of the above model, we assume that the distribution of the cash flows is according to an exponential distribution:

$$
f(s)=\frac{1}{\gamma} e^{-s / \gamma}, s \geq 0
$$

for some $\gamma>0$. We also consider the limiting case $\gamma=0$, which represents the case when all cash flows occur at the time $\tau+\tau_{B}$ (i.e. $F(s)=\delta_{0}(s)$ ). When $\gamma>0$ we have

$$
\int_{0}^{\infty} e^{-q s} \mathrm{~d} F(s)=\int_{0}^{\infty} e^{-q s} \frac{1}{\gamma} e^{-\frac{1}{\gamma} s} d s=\frac{1}{\gamma q+1} .
$$

One way of interpreting the parameter $\gamma$ is that it reflects the competitiveness of the market for the product the investment generates. In a market with many competitors, we expect competition to make the cash flows occurring later than in a less competitive market. As $\gamma$ increases, the cash flows are occurring later, making the interpretation that the higher the value on $\gamma$, the more competitive the market is, possible. With this specification of $F$, we have

$$
k=e^{q \delta} \cdot(1+q / \lambda) \cdot(1+q \gamma) .
$$

In Tables 1, 2 and 3 numerical values of the factor $k$ is presented. The value $\lambda=\infty$ represents the case when there is no exponentially distributed waiting time after $\tau+\delta$. In all cases $\delta=2$.

With this specification of delay distribution $F$ we have

$$
k=e^{q \delta+\ln (1+q / \lambda)+\ln (1+q \gamma)} \approx e^{q(\delta+1 / \lambda+\gamma)} .
$$

Table 1 The factor $k$ when the yield is $q=0.01$

\begin{tabular}{lllll}
\hline$\gamma \backslash \lambda$ & $\infty$ & 10 & 5 & 1 \\
\hline 0 & 1.020 & 1.021 & 1.022 & 1.030 \\
0.5 & 1.025 & 1.026 & 1.027 & 1.036 \\
1 & 1.030 & 1.031 & 1.032 & 1.041 \\
5 & 1.071 & 1.072 & 1.073 & 1.082 \\
\hline
\end{tabular}


Table 2 The factor $k$ when the yield is $q=0.02$

\begin{tabular}{lllll}
\hline$\gamma \backslash \lambda$ & $\infty$ & 10 & 5 & 1 \\
\hline 0 & 1.041 & 1.043 & 1.045 & 1.062 \\
0.5 & 1.051 & 1.053 & 1.055 & 1.072 \\
1 & 1.062 & 1.064 & 1.066 & 1.083 \\
5 & 1.145 & 1.147 & 1.149 & 1.168 \\
\hline
\end{tabular}

\begin{tabular}{lllll}
\hline$\gamma \backslash \lambda$ & $\infty$ & 10 & 5 & 1 \\
\hline 0 & 1.105 & 1.111 & 1.116 & 1.160 \\
0.5 & 1.133 & 1.138 & 1.144 & 1.189 \\
1 & 1.160 & 1.166 & 1.172 & 1.218 \\
5 & 1.381 & 1.388 & 1.395 & 1.451 \\
\hline
\end{tabular}

Table 3 The factor $k$ when the yield is $q=0.05$

Hence, for a fixed level of the sum of $\delta+1 / \lambda+\gamma$, so we expect that the higher the yield $q$, the higher is the value of the factor $k$. This is also seen in the tables to be the case.

\section{Conclusion}

The fact that cash flows occur later than at the time at which the investment is decided on, and specifically that they are spread out in time after the time at which cash flows generated by the investment are beginning to occur, is often neglected in models of irreversible investments. We combine two existing models of investment lags to obtain a flexible, yet simple, way of modelling and analyzing the effects of investment lags. Furthermore, the main focus of this paper is to address the investment problems when the cash flows generated by an investment can be spread out in time. The aim has been to present a simple, yet fairly general, model. One advantage with this approach is that it is possible to write the optimal stopping problem in a form that makes it straightforward to apply standard techniques from optimal stopping theory to solve the problem.

The main structure of the modelling framework was the following: After the time $\tau$ at which the investment is initiated, and when there can be a cash flow, there passes a random time $\tau_{B}$ during which there are no cash flows. After the time $\tau+\tau_{B}$ the investment is starting to generate cash flows. What distinguishes our approach is that there can be cash flows during the interval $\left[\tau+\tau_{B}, \infty\right)$, not only at time $\tau+\tau_{B}$. These cash flows are distributed according to the deterministic distribution $F$, so the randomness with respect to time ends at $\tau+\tau_{B}$. This is one limitation present in the framework, and something that future research could generalise (e.g. to allow for a random measure determining the distribution of the cash flows). Another limitation is that the only part of the model that the investor can choose is the time $\tau$ at which the investment is initiated. It would be interesting to extend this part of the model to 
include for the investor to control e.g. the distribution, but not the exact length, of $\tau_{B}$ or to choose the distribution of the cash flows after $\tau+\tau_{B}$.

As a concrete example, we considered the standard optimal investment problem with a constant investment cost from McDonald and Siegel (1986), and study it when we include both a time-to-build and when there are lags in the cash flows. In this case, the solution is a modified version of the solution to the standard problem, and this modified solution depends on a single parameter. This parameter has a multiplicative decomposition, where each part of the decomposition is aligned to one specific property of the model.

Funding Information Open access funding provided by Royal Institute of Technology. We thank the research project Housing 2.0 (Bostad 2.0) for financial support.

Data availability This article has no associated data.

\section{Declarations}

Conflict of interest The authors declare that there are no conflicts of interest.

Open Access This article is licensed under a Creative Commons Attribution 4.0 International License, which permits use, sharing, adaptation, distribution and reproduction in any medium or format, as long as you give appropriate credit to the original author(s) and the source, provide a link to the Creative Commons licence, and indicate if changes were made. The images or other third party material in this article are included in the article's Creative Commons licence, unless indicated otherwise in a credit line to the material. If material is not included in the article's Creative Commons licence and your intended use is not permitted by statutory regulation or exceeds the permitted use, you will need to obtain permission directly from the copyright holder. To view a copy of this licence, visit http://creativecommons.org/licen ses/by/4.0/.

\section{References}

Aguerrevere FL (2003) Equilibrium investment strategies and output price behavior: a real-options approach. Rev Financ Stud 16(4):1239-1272. https://doi.org/10.1093/rfs/hhg041

Alvarez LHR, Keppo J (2002) The impact of delivery lags on irreversible investment under uncertainty. Eur J Oper Res 136:173-180

Armerin F, Song H-S (2018) Valuation of real options in incomplete models - an implied yield approach. Fuzzy Econ Rev 23(1): 19-32. https://doi.org/10.25102/fer.2018.01.02

Balliauw M (2020) Time to build: a real options analysis of port capacity expansion investments under uncertainty. https://doi.org/10.1016/j.retrec.2020.100929

Bar-Ilan A, Strange WC (1996) Investment Lags. Am Econ Rev 86(3):610-622

Bar-Ilan A, Sulem A, Zanello A (2002) Time-to-build and capacity choice. J Econ Dyn Control 26:69-98

Dixit AK, Pindyck RS (1994) Investment under uncertainty. Princeton University Press, Princeton

Grenadier SR (1995) The persistence of real estate cycles. J Real Estate Financ Econ 95-119

Grenadier SR (2000) Equilibrium -with time-to-build: a real options approach. Project flexibility, agency, and competition: new developments in the theory and applications of real options. Oxford University Press, Oxford

Jeon H (2021a) Investment and financing decisions in the presence of time-to-build. Eur J Oper Res 288(3):1068-1084. https://doi.org/10.1016/j.ejor.2020.06.034

Jeon $\mathrm{H}$ (2021b) Investment timing and capacity decisions with time to build in a duopoly market. J Econ Dyn Control 122. https://doi.org/10.1016/j.jedc.2020.104028

Jeanblanc M, Yor M, Chesny M (2009) Mathematical methods for financial markets. Springer, London 
Kalouptsidi M (2014) Time to build and fluctuations in bulk shipping. Am Econ Rev 104(2):564-608

Koeva P (2000) The Facts about time-to-build. IMF Working Paper

Lempa J (2012) Optimal stopping with random exercise lag. Math Methods Oper Res 75:273-286. https://doi.org/10.1007/s00186-012-0384-7

Lempa J (2020) Some results on optimal stopping under phase-type distributed implementation delay. Math Methods Oper Res. https://doi.org/10.1007/s00186-019-00694-6

MacDougall SL, Pike RH (2003) Consider your options: changes to strategic value during implementation of advanced manufacturing technology. Omega 1-15

Majd S, Pindyck RS (1987) Time to build, option value, and investment decisions. J Financ Econ 18:2-27

Margsiri W, Mello AS, Ruckes ME (2003) A dynamic analysis of growth via acquisition. Rev Financ, pp. 635-671. https://doi.org/10.1093/rof/rfn015

Marmer V, Slade ME (2018) Investment and uncertainty with time to build: evidence from entry into U.S. copper mining. J Econ Dyn Control 95:233-254. https://doi.org/10.1016/j.jedc.2018.09.001

McDonald R, Siegel D (1986) The Value of Waiting to Invest. Q J Econ 101(4):707-727

Oh H, Yoon C (2020) Time to build and the real-options channel of residential investment. J Financ Econ 135:255-269. https://doi.org/10.1016/j.jfineco.2018.10.019

Øksendal B (2005) Optimal stopping with delayed information. Stochast Dyn 5(2):271-280

Pacheco-de-Almeida G, Zemsky P (2003) The effect of time-to-build on strategic investment under uncertainty. Rand J Econ 34(1):166-182

Sarkar S, Zhang C (2013) Implementation lag and the investment decision. Econ Lett 119:136-140

Sarkar S, Zhang C (2015) Investment policy with time-to-build. J Bank Financ 55:142-156

Taschini L (2021) Flexibility premium of emissions permits. J Econ Dyn Control. https://doi.org/10. 1016/j.jedc. 2020.104013 